\title{
Análisis de la satisfacción del visitante de cruceros desde las perspectivas evaluativa y emocional
}

\author{
María de Jesús Moo Canul* \\ Universidad de Quintana Roo \\ Ángel Quintero Sánchez \\ Universidad Tecnológica de la Mixteca \\ Vanessa Liliana Velázquez Domínguez \\ Tania Delgado Hernández \\ Instituto Politécnico Nacional
}

\section{Resumen}

En México, el Centro de Estudios Superiores de Turismo y la Secretaría de Turismo, junto con otros centros de investigación, han realizado estudios relacionados con el turismo y, en particular, con la satisfacción del visitante. Sin embargo, los resultados que se presentan en sus páginas oficiales son transversales y se integran con el perfil del turista, lo que los convierte en un instrumento sumamente extenso que, para el caso de los visitantes de cruceros, limita la posibilidad de aplicar fielmente el instrumento. El objetivo de este artículo es analizar la satisfacción del visitante que llega a un destino de cruceros con base en las perspectivas evaluativa y emocional. La metodología consistió en revisar la literatura del concepto, para comprender el contexto de la temática así como los enfoques que se abordan, incorporando estas dos dimensiones en un cuestionario, el cual fue aplicado a 385 visitantes que llegaron a Cozumel en cruceros, en la segunda y la tercera semanas de julio de 2013. Se identificó que un grupo considerable no consume los servicios de alimentación, pues estos son parte de los que se ofrecen en el crucero. Los hallazgos permiten ofrecer un panorama de la situación de esta temática y la posibilidad de replicarla de manera longitudinal.

\section{Palabras clave}

Satisfacción, visitante, satisfacción evaluativa, satisfacción emocional.

Recibido: 11/06/2014 · Aceptado: 05/09/2014

*Correo electrónico: mariajmc@uqroo.edu.mx; quinterosanchezangel@hotmail.com; nessalivelzquez@gmail.com; danyherforever@gmail.com 


\title{
Analysis of cruise ship visitors from an evaluative and emotional perspective
}

\author{
María de Jesús Moo Canul* \\ Universidad de Quintana Roo \\ Ángel Quintero Sánchez \\ Universidad Tecnológica de la Mixteca \\ Vanessa Liliana Velázquez Domínguez \\ Tania Delgado Hernández \\ Instituto Politécnico Nacional
}

\begin{abstract}
The Center for Tourism Studies (Cestur) and the Tourism Secretariat (Sedetur) as well as other research centers in Mexico, have developed studies related in particular to visitor satisfaction. The results that are presented on their web pages are of a transversal character and are integrated with the visitor profile generating a rather extensive database limiting it's application. The objective of this study is to analyze visitor satisfaction in a cruise ship destination from an evaluative and emotional perspective. The methodology consisted of a extensive bibliographical review to understand the focus applied to the concept as well as to the context including both dimensions in a survey that was applied the second and third weeks in July 2013 to 385 visitors. Data confirm visitor satisfaction from an evaluative and emotional perspective and identify that a vast majority of visitors do not consume food services since these are offered onboard. Results allow the presentation of an outlook of the subject and the possibility of replicating it in a longitudinal model.
\end{abstract}

\section{KEY WORDS}

Satisfaction, visitor, evaluative satisfaction, emotional satisfaction.

*E-mail: mariajmc@uqroo.edu.mx; quinterosanchezangel@hotmail.com; nessalivelzquez@ gmail.com; danyherforever@gmail.com 


\section{Introducción}

El concepto satisfacción del consumidor puede analizarse desde diversas perspectivas, incluyendo la medición de la calidad de los servicios, la cual es útil para determinar las estrategias que deben emplearse para lograr la satisfacción del cliente (Brown y Swartz, 1989; Parasuraman, Zeithaml y Berry, 1989; Rudie y Wansley, 1985; Thompson, DeSouza y Gale, 1985, cit. por Cronin y Taylor, 1992). Es decir, "la entrega de mayores niveles de calidad del servicio es la estrategia que, cada vez más, se ofrece como una clave para que los esfuerzos de los proveedores de servicios los posicionen más eficazmente en el mercado" (Cronin y Taylor, 1992: 55).

Las organizaciones deben buscar la manera de saber qué es lo que los clientes realmente necesitan; en este sentido, tanto Jacksons como Anderson y Narus (1991, cit. por Garbarino y Johnson, 1999) afirman que "las organizaciones deben analizar las posiciones que simultáneamente podrían necesitar en la comercialización transaccional y relacional, ya que no todos los clientes quieren la misma relación de trabajo” (p. 70).

Para responder a este requerimiento de información, las organizaciones recurren a distintos métodos y procedimientos para medir la satisfacción de los clientes, considerando que "los árbitros definitivos de la satisfacción son los propios clientes” (Hill, Brierley y MacDougall, 2001: 9); razón por la cual, también sostienen estos autores, "solo en los niveles más elevados de satisfacción del cliente existe una fuerte correlación entre lealtad y satisfacción” (2001: 11).

Una encuesta que mida la satisfacción del cliente puede proporcionar datos muy precisos y resaltar las áreas en las que los clientes están menos contentos, pero no puede resolver los problemas, mejorar el servicio ni aumentar la satisfacción de aquellos (Hill, Brierley y MacDougall, 2001: 12). Por lo tanto, para construir la marca del destino, resulta importante analizar diferentes modelos de medición de la satisfacción del turista, además de examinar la satisfacción respecto de los principales sectores que se ven beneficiados por la actividad turística. 


\section{Antecedentes}

Las investigaciones realizadas para medir la satisfacción del cliente han sugerido que esta puede ser explicada desde varios enfoques, como los de tipo evaluativo y afectivo (Cronin, Brady y Hult, 2000; Evrard y Aurier, 1994; Oliver, 1997; Swan y Trawick, 1993; Westbrook y Oliver, 1991; Izard, 1977). Tales estudios no han sido originalmente planteados para la actividad turística, pero hay documentos que los aplican para tratar de explicar la satisfacción del turista (Alegre y Garau, 2010; Rodríguez y San Martín, 2008; Yu y Goulden, 2006; Reynolds y Braithwaite, 2001; Latu y Everett, 2000; entre otros).

Algunas investigaciones realizadas en años recientes defienden que existe una dualidad al realizar un juicio de valor respecto de la experiencia vivida en el consumo turístico. De acuerdo con su análisis de las corrientes cognitivo-afectivas, Femenía (2011) precisa que hay investigaciones que apoyan el enfoque cognitivo-afectivo de la satisfacción del consumidor, derivadas de la interpretación y evaluación que realiza el consumidor al formar sus emociones, y estipula que estas pueden ser deliberadas y conscientes o autonómicas e inconscientes (Bagozzi, 1999, cit. por Femenía, 2011), del mismo modo que alude a lo que Oliver denomina el razonamiento causal (1989, cit. por Femenía, 2011). Estas orientaciones permiten asumir que existe una dualidad (cognitiva y afectiva) en la percepción de la experiencia del turista.

En México, la Secretaría de Turismo (Sectur) -a través del Centro de Estudios Superiores en Turismo (Cestur), en la actualidad Instituto para la Competitividad Turística- ha efectuado en los últimos diez años el estudio Perfil y Grado de Satisfacción de los Turistas, a fin de conocer el nivel de satisfacción de estos en los principales destinos turísticos del país (Cestur, 2012).

El Cestur (2012) "ha diseñado una estrategia de transferencia del conocimiento a los destinos turísticos para el mejor aprovechamiento de la información, de tal manera que sean los propios actores locales los encargados de emprender los estudios", y elaboró un instrumento para que sea aplicado con este fin; el análisis es llevado a cabo en varias dimensiones "la imagen y acceso del destino, servicios y atractivos turísticos conocidos por el turista, experiencia de viaje y costo. La recomendación se considera factor resultante de la satisfacción” (Cestur, 2012). El instrumento consta de ocho secciones y 29 preguntas con sus respectivas opciones a calificar, lo cual dificulta su aplicación para 
evaluar la satisfacción de visitantes que llegan en cruceros, debido a que disponen de poco tiempo para su visita en el puerto y lo ocupan para actividades de recreación.

El principal destino de crucero en México es la isla de Cozumel, donde año con año se reciben millones de turistas que desembarcan y pasean como parte de la ruta por el Caribe mexicano. En la gráfica 1 puede observarse que Cozumel recibió 63.4\% de los pasajeros que llegaron a México durante 2013, lo cual representa a más de la mitad de pasajeros que arribaron al país en crucero.

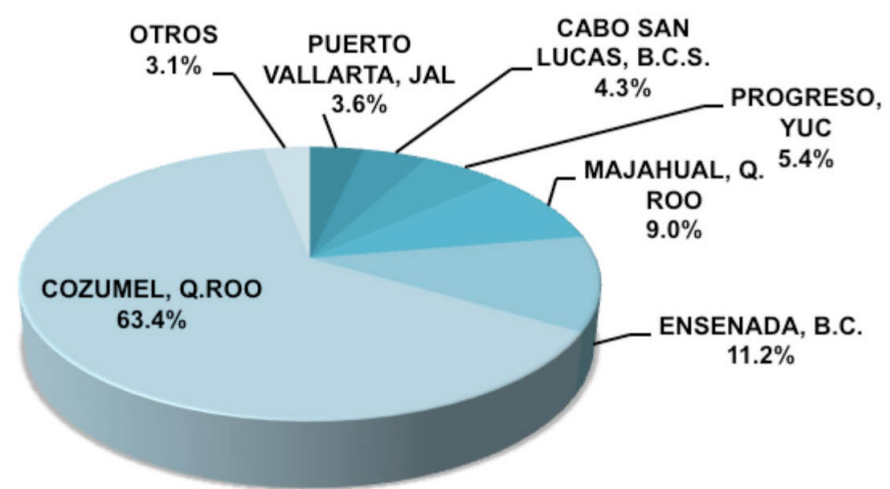

Fuente: Informe estadístico mensual de la Dirección General de Puertos-Secretaría de Comunicaciones y Transportes (2013).

GRÁFICA 1. Distribución del movimiento de cruceros (pasajeros) enero-diciembre 2013

La importancia del destino también radica en el monto de divisas que ingresan anualmente al país por la actividad turística. En 2012, 38.9\% del total de divisas que entraron a México fueron por este concepto y, de enero a diciembre de 2013, en Quintana Roo se obtuvo un acumulado 7577.92 millones de dólares de derrama económica (Sedetur, 2013), equivalentes a una tercera parte de lo que recibe la república mexicana; en consecuencia, es posible constatar la importancia económica de este estado en el sector turismo nacional.

Como ya se dijo, los visitantes de cruceros disponen de poco tiempo para bajar y visitar los destinos, en escasas ocasiones se percatan de lo que el destino ofrece en su totalidad, y su experiencia con los servicios que ahí se brindan se limita al impacto que las pocas horas en las que tuvieron contacto pudieron 
registrar en su mente; esto significa que los sitios que tocan los cruceros tienen menos de un día para satisfacer las expectativas de los visitantes.

\section{Metodología}

El objetivo de este trabajo es analizar dos escalas que se utilizan para evaluar la satisfacción del turista revisando cómo está conformada, qué investigaciones se han realizado, cómo se presentan los datos descriptivos generales y concretando un análisis factorial de segundo orden y el sistema de ecuaciones estructurales, de manera tal que sea posible tener una idea de la satisfacción de los visitantes de cruceros.

La investigación es cuantitativa, y utiliza un muestreo no probabilístico de conveniencia. Los resultados se exponen en la primera parte con estadísticos descriptivos, identificando los ítems señalados en las escalas evaluativa y emocional. Estos ítems se integraron en un solo instrumento, que fue aplicado en la segunda y tercera semanas de julio de 2013 a los visitantes que arribaron a Cozumel vía cruceros. No debe olvidarse que Cozumel cuenta con tres terminales marítimas para recibir a los visitantes de cruceros: Puerta Maya, Punta Langosta y SSA International Cruise Terminal. Las encuestas se aplicaron en las dos primeras, en razón de la cantidad de navieras que desembarcaron en esas terminales en las semanas señaladas.

Los tres ítems de la escala evaluativa fueron tomados de la versión original del estudio de Cronin, Brady y Hult (2000), que a su vez fue inspirada en la investigación de Oliver (1997). Los ítems se midieron en una escala de Likert de nueve puntos, y se busca determinar el grado de lo que el turista cree en cuanto a la decisión que tomó para adquirir dicho servicio.

CUADRo 1. Ítems para medir la satisfacción desde la perspectiva evaluativa

1. My choice to purchase the service was a wise one

2. I think that I did the right thing when I purchase this service

3. This facility is exactly what I needed for this service 
Para la medición emocional se utilizaron los ítems sugeridos por Cronin, Brady y Hult (2000), quienes adaptaron las escalas usadas por Westbrook y Oliver (1991), empleando la escala de disociación de experiencias (dissociative experiences scale II, DES-II). Este enfoque utiliza una escala de Likert de nueve niveles, y busca reflejar cómo se siente el turista en relación con los servicios recibidos (con la advertencia de que no existen respuestas malas o buenas). Cinco ítems, presentados en el cuadro 2, conforman esta escala.

\section{Cuadro 2. Ítems para medir la satisfacción desde} la perspectiva emocional

\section{Interest \\ 2. Enjoyment \\ 3. Surprise \\ 4. Anger \\ 5. Shame/shyness}

Fuente: Cronin, Brady y Hult (2000).

Se prescindió de los dos últimos ítems de la escala original, que no se ajustaban al objetivo de esta investigación, es decir, evaluar emocionalmente los dos principales servicios ofrecidos en el destino turístico: alimentación y servicios turísticos, además de la percepción general de los atractivos del destino. En cambio, se incluyeron los tres ítems útiles para cubrir el propósito aquí planteado, y establecer los elementos coincidentes de la propuesta metodológica del Cestur (2012), descrita en el cuadro 3, y los de medición de factores de la escala de Kozak y Rimmington (2000), que considera cuatro aspectos (cuadro 4).

Con todo, se presentó un considerable número de casos, con valores faltantes en ambas escalas, y no fue posible tener un buen ajuste en el modelo estructural propuesto al inicio, pues se encontró que los encuestados no suelen utilizar los servicios de excursión/turísticos y/o de alimentación, por lo que se precisó recurrir a los ítems que evalúan únicamente su satisfacción respecto de los atractivos del destino. 
Cuadro 3. Aspectos para medir la satisfacción del turista de acuerdo con el Cestur

\begin{tabular}{ll}
\hline Imagen y acceso del destino & $\begin{array}{l}\text { Servicios y atractivos turísticos conocidos por el } \\
\text { turista }\end{array}$ \\
Experiencia de viaje & Costo \\
\hline
\end{tabular}

Fuente: Cestur (2012).

Cuadro 4. Aspectos para medir la satisfacción del turista de acuerdo con Kozak y Rimmington

\begin{tabular}{ll}
\hline Destination attractiveness & Tourist attractions and facilities \\
Availability of English language & Facilities and services at the destination airport \\
\hline
\end{tabular}

Fuente: Kozak y Rimmington (2000).

Ciertos análisis se pronuncian en favor de hacer modelos estructurales a partir de muestras pequeñas (Hoyle, 1995), y usualmente recomiendan una muestra de entre 100 y 150 encuestados, mientras que otros investigadores afirman que se requiere una muestra mayor, por ejemplo 200 (Hoogland y Boomsma, 1998). Adicionalmente, estudios de simulación revelan que, con datos con una distribución normal, sin datos faltantes y para un análisis factorial confirmatorio simple, un tamaño de muestra de 150 es recomendable (Muthén y Muthén, 2002).

Teniendo en cuenta lo anterior, los hallazgos de este artículo corresponden a la aplicación de 385 cuestionarios. En la primera sección los resultados se exponen desde un punto de vista general, para comparar los resultados en cada una de las escalas como parte de la comprobación empírica de las teorías de la satisfacción emotiva y evaluativa; después se ofrece el análisis factorial confirmatorio de segundo orden y el modelo estructural probado.

\section{Análisis de los principales hallazgos}

Durante el periodo de aplicación de la encuesta (segunda y tercera semanas de julio de 2013), quienes arribaron a Cozumel vía cruceros lo hicieron princi- 
palmente en la línea naviera de Carnival Cruise Line (16 de 19 embarcaciones según datos estadísticos de la Administración Portuaria Integral de Quintana Roo, 2013). Es importante anotar que, como los resultados corresponden sobre todo a esta línea naviera, en ningún caso se pretende extender inferencias hacia las demás navieras.

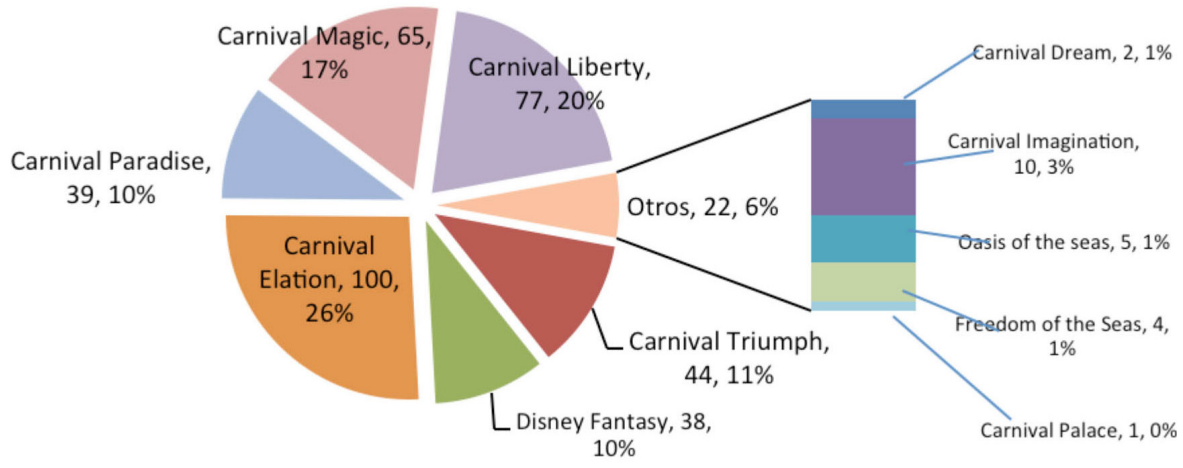

Fuente: Elaboración propia con base en las encuestas $(\mathrm{n}=385)$.

GráfICA 2. Número de visitantes encuestados por línea naviera

En la gráfica 2 se señalan las embarcaciones encuestadas; ocho de las 11 embarcaciones corresponden a la Carnival Cruise Line, una a la Disney Company y una a la Royal Caribbean International. El cuadro 5 describe el perfil de los visitantes de cruceros entrevistados.

Con base en lo expuesto en el cuadro 5, puede resumirse el perfil de los visitantes encuestados: son personas que viajan en familia, en su mayoría mujeres, sus principales ocupaciones son profesional/técnico o empleado y son predominantemente universitarios. 
Cuadro 5. Perfil de los visitantes de cruceros encuestados

\begin{tabular}{lr} 
Característica & Porcentaje \\
Viaja con & \\
Solo & 4.7 \\
Familia & 67.0 \\
Amigos & 7.0 \\
Pareja & 14.5 \\
Compañeros de trabajo & 3.1 \\
Grupo & 3.6 \\
Sexo & \\
Hombre & 41.6 \\
Mujer & 58.4 \\
Ocupación & \\
Estudiante & 15.6 \\
Empleado & 21.0 \\
Empleado de gobierno & 5.7 \\
Profesional/técnico & 22.6 \\
Otro & 10.4 \\
Esposa & 5.7 \\
Director/ejecutivo & 1.8 \\
Propietario de negocio & 7.0 \\
Retirado & 10.1 \\
Grado académico & \\
Educación media básica & 1.0 \\
Preparatoria & 19.7 \\
Universitario & 48.3 \\
Profesional & 1.8 \\
Especialidad & \\
Maestría o doctorado & \\
Posdoctorado & \\
Otro & \\
\hline & \\
\hline
\end{tabular}

Resultados de la escala de satisfacción evaluativa

A fin de establecer la fiabilidad de la escala, se obtuvo un alfa de Cronbach de 0.930, considerando que, típicamente, una alfa mayor a 0.70 es deseable (Nunnally y Bernstein, 1994). 


\section{Cuadro 6. Estadísticos descriptivos de la satisfacción evaluativa}

\begin{tabular}{|c|c|c|c|c|c|c|}
\hline \multirow{2}{*}{ Ítems en idioma inglés } & \multicolumn{3}{|c|}{$\mathrm{N}$} & \multirow[b]{2}{*}{ Máximo } & \multirow[b]{2}{*}{ Media } & \multirow[b]{2}{*}{ Desv. típ. } \\
\hline & Válido & Perdido & Mínimo & & & \\
\hline $\begin{array}{l}\text { My choice to purchase the } \\
\text { food service was a wise } \\
\text { one }\end{array}$ & 309 & 76 & 2 & 9 & 7.98 & 1.302 \\
\hline $\begin{array}{l}\text { I think that I did the right } \\
\text { thing when I purchase this } \\
\text { food service }\end{array}$ & 303 & 82 & 2 & 9 & 8.01 & 1.231 \\
\hline $\begin{array}{l}\text { This facility is exactly } \\
\text { what I needed for this food } \\
\text { service }\end{array}$ & 305 & 80 & 3 & 9 & 8.02 & 1.207 \\
\hline $\begin{array}{l}\text { My choice to purchase the } \\
\text { excursion/tourist service } \\
\text { was a wise one }\end{array}$ & 321 & 64 & 1 & 9 & 8.11 & 1.353 \\
\hline $\begin{array}{l}\text { I think that I did the right } \\
\text { thing when I purchase this } \\
\text { excursion/tourist service }\end{array}$ & 316 & 69 & 1 & 9 & 8.14 & 1.320 \\
\hline $\begin{array}{l}\text { This facility is exactly } \\
\text { what I needed for this } \\
\text { excursion/tourist service }\end{array}$ & 319 & 66 & 1 & 9 & 8.14 & 1.298 \\
\hline $\begin{array}{l}\text { My choice to visit the } \\
\text { destination was a wise } \\
\text { one }\end{array}$ & 371 & 14 & 2 & 9 & 8.27 & 1.151 \\
\hline $\begin{array}{l}\text { I think that I did the right } \\
\text { thing when I visit the } \\
\text { destination }\end{array}$ & 367 & 18 & 2 & 9 & 8.26 & 1.215 \\
\hline $\begin{array}{l}\text { This facility is exactly } \\
\text { what I needed for my visit } \\
\text { to Cozumel }\end{array}$ & 366 & 19 & 1 & 9 & 8.19 & 1.242 \\
\hline \multicolumn{7}{|c|}{ Estadísticos de fiabilidad } \\
\hline Alfa de Cronbach & \multicolumn{5}{|c|}{ Alfa de Cronbach basada en los elementos tipificados } & $\begin{array}{c}\mathrm{N} \text { de } \\
\text { elementos }\end{array}$ \\
\hline 0.930 & \multicolumn{5}{|c|}{0.932} & 9 \\
\hline
\end{tabular}

Fuente: Elaboración propia con base en las encuestas. Escala de Likert, donde 1 significa strongly disagree y 9 strongly agree. 
Evaluamos tres aspectos: servicios de alimentación, servicios de excursión/ turísticos y los atractivos del destino, en virtud de que son las actividades predominantes de los visitantes de cruceros en Cozumel.

Los ítems de la escala para la parte evaluativa (mi decisión de comprar este servicio fue sabia; yo pienso que hice lo correcto cuando compré este servicio; este servicio es exactamente lo que necesitaba de él) se tomaron de la escala original del estudio de Cronin, Brady y Hult (2000) quienes, como ya se dijo, la recuperaron de la investigación de Oliver (1997). Esta escala sugiere calificar los ítems de acuerdo con la escala de Likert en nueve niveles, donde 1 es strongly disagree y 9 strongly agree.

En cuanto a los servicios de alimentación, se observó que en promedio los encuestados lo califican satisfactorio, otorgándole calificación de 8.0; pero es menester señalar que $20.6 \%$ de los encuestados no respondió este apartado.

En materia de servicios de excursión y turísticos, le asignaron en promedio calificaciones positivas (8.13), pero también resalta un porcentaje significativo que no evaluó esta sección (17.2\%)

Por lo que toca a la satisfacción con el destino, al calificarlo, en general consideran que sí cumplió sus expectativas, y en promedio a su satisfacción le otorgan un puntaje de 8.24. Solo $4.4 \%$ no evaluó este aspecto.

Resultados de la escala de satisfacción emocional

Al comprobar la fiabilidad de la escala que evalúa la satisfacción emocional, se obtuvo un alfa de Cronbach de 0.905.

Para tasar la satisfacción desde el enfoque emocional se utilizaron los ítems sugeridos por Cronin, Brady y Hult (2000), los cuales, como ya se estableció líneas arriba, adaptaron las escalas usadas por Westbrook y Oliver (1991): DEs-II. Nuevamente se pidió a los encuestados que valoraran los servicios de alimentación, los servicios de excursión/turísticos y los atractivos del destino, a partir de los tres ítems que conforman la escala DEs-II: interest, enjoyment y surprise.

Al referirse a los servicios de alimentación en Cozumel, los encuestados calificaron su satisfacción con 7.68 en promedio, es decir, los juzgan como algo agradable. Sin embargo, cuando se les inquirió si les pareció sorprendente, se obtuvo una respuesta con mayor dispersión de los datos, ya que su desviación típica es de 1.772. Además, otra vez se encontró que 18.96 \% no emitió opinión. 
Cuadro 7. Estadísticos descriptivos de la satisfacción emocional

\begin{tabular}{|c|c|c|c|c|c|c|}
\hline \multirow{2}{*}{ Ítems en idioma inglés } & \multicolumn{2}{|c|}{$\mathrm{N}$} & \multirow[b]{2}{*}{ Mínimo } & \multirow[b]{2}{*}{ Máximo } & \multirow[b]{2}{*}{ Media } & \multirow[b]{2}{*}{ Desv. típ. } \\
\hline & Válido & Perdido & & & & \\
\hline Food services in Cozumel is interest & 314 & 71 & 1 & 9 & 7.71 & 1.576 \\
\hline Food services in Cozumel is enjoyment & 313 & 72 & 1 & 9 & 7.92 & 1.408 \\
\hline Food services in Cozumel is surprise & 309 & 76 & 1 & 9 & 7.43 & 1.772 \\
\hline $\begin{array}{l}\text { Services excursion/tourist in Cozumel } \\
\text { is interest }\end{array}$ & 326 & 59 & 1 & 9 & 8.02 & 1.435 \\
\hline $\begin{array}{l}\text { Services excursion/tourist in Cozumel } \\
\text { is enjoyment }\end{array}$ & 328 & 57 & 1 & 9 & 8.05 & 1.365 \\
\hline $\begin{array}{l}\text { Services excursion/tourist in Cozumel } \\
\text { is surprise }\end{array}$ & 317 & 68 & 1 & 9 & 7.64 & 1.739 \\
\hline Destination attractiveness is interest & 379 & 6 & 1 & 9 & 8.17 & 1.223 \\
\hline $\begin{array}{l}\text { Destination attractiveness is } \\
\text { enjoyment }\end{array}$ & 376 & 9 & 2 & 9 & 8.21 & 1.191 \\
\hline Destination attractiveness is surprise & 368 & 17 & 1 & 9 & 7.79 & 1.697 \\
\hline \multicolumn{7}{|c|}{ Estadísticos de fiabilidad } \\
\hline Alfa de Cronbach & \multicolumn{5}{|c|}{$\begin{array}{c}\text { Alfa de Cronbach basada en los elementos } \\
\text { tipificados }\end{array}$} & $\begin{array}{l}\mathrm{N} \text { de } \\
\text { elementos }\end{array}$ \\
\hline 0.905 & \multicolumn{5}{|c|}{0.911} & 9 \\
\hline
\end{tabular}

Fuente: Elaboración propia con base en las encuestas. Escala de Likert donde 1 significa Strongly disagree y 9 Strongly agree $(\mathrm{n}=385)$.

En cuanto a los servicios de excursión y turísticos, les concedieron en promedio un puntaje de 7.90, calificación aún positiva. Aquí, se registró una mayor distribución de las calificaciones otorgadas para el ítem asociado con su perspectiva de lo sorprendente que pudo llegar a ser el servicio, con una desviación típica fue de 1.739. Del total de las encuestas, 15.9 \% no respondió esta parte.

En términos generales, desde la perspectiva emocional, la satisfacción de los atractivos del destino obtiene mejores resultados: le otorgaron, en promedio, 8.056, y con menores desviaciones típicas, además de que solo $2.77 \%$ no contestó este rubro. 


\section{Análisis factorial confirmatorio (AFC)}

El cuestionario fue diseñado con apego a los aspectos por evaluar en el destino, teniendo en consideración los que sugieren el Cestur (2012) y Kozak y Rimmington (2000), y utilizando las escalas sugeridas en las teorías de la satisfacción emocional y evaluativa (Cronin, Brady y Hult, 2000), siguiendo un modelo de análisis factorial confirmatorio de segundo orden y sistemas de ecuaciones estructurales, capturado y procesado en el programa estadístico sPss, versión 19 y MPlus versión 7, el cual permite realizar análisis con valores perdidos.

\section{Cuadro 8. Resultados del análisis factorial confirmatorio}

\begin{tabular}{|c|c|c|c|c|c|}
\hline Hipótesis & Relación estructural planteada & $\lambda$ Std. & \multicolumn{3}{|c|}{ Indicadores de bondad de ajuste } \\
\hline $\mathrm{H}_{1}$ & $\begin{array}{l}\text { Satisfacción del visitante de cruceros- } \\
\text { satisfacción evaluativa }\end{array}$ & 0.841 & \multirow{2}{*}{\multicolumn{3}{|c|}{$\begin{array}{l}\text { CFI }=0.975 ; \text { TLI }=0.947 \\
\text { RMSEA }=0.142 ; \text { SRMR }=0.025 \\
\text { Chi-Square Test of Model Fit }= \\
60.844 \text { (7gl), P-Value }=0.00\end{array}$}} \\
\hline $\mathrm{H}_{2}$ & $\begin{array}{l}\text { Satisfacción del visitante de cruceros- } \\
\text { satisfacción emocional }\end{array}$ & 0.794 & & & \\
\hline \multicolumn{6}{|c|}{ Descripción } \\
\hline \multicolumn{3}{|c|}{ (F1) Satisfacción evaluativa (ítems en idioma inglés) $\lambda=0.930$} & $\lambda$ Std. & Err.std. & Valor de $\mathrm{P}$ \\
\hline P7 & \multicolumn{2}{|c|}{ My choice to visit the destination was a wise one } & 0.950 & 0.007 & $<0.001$ \\
\hline P8 & \multicolumn{2}{|c|}{$\begin{array}{l}\text { I think that I did the right thing when I visit the } \\
\text { destination }\end{array}$} & $0.978^{\#}$ & 0.005 & $<0.001$ \\
\hline P9 & \multicolumn{2}{|c|}{$\begin{array}{l}\text { This facility is exactly what I needed for my visit to } \\
\text { Cozumel }\end{array}$} & 0.906 & 0.011 & $<0.001$ \\
\hline \multicolumn{3}{|c|}{ (F2) Satisfacción emocional (ítems en idioma inglés) $\lambda=0.905$} & $\lambda$ Std. & Err.std. & Valor de $\mathrm{P}$ \\
\hline P16 & \multicolumn{2}{|l|}{ Destination attractiveness was interest } & 0.854 & 0.019 & $<0.001$ \\
\hline P17 & \multicolumn{2}{|l|}{ Destination attractiveness was enjoyment } & 0.971 & 0.014 & $<0.001$ \\
\hline P18 & \multicolumn{2}{|l|}{ Destination attractiveness was surprise } & $0.686^{*}$ & 0.03 & $<0.001$ \\
\hline \multicolumn{6}{|c|}{ (G) Factorial de Segundo Orden } \\
\hline F1 & \multicolumn{2}{|l|}{ Satisfacción evaluativa } & 0.841 & 7.382 & 0.909 \\
\hline $\mathrm{F} 2$ & \multicolumn{2}{|l|}{ Satisfacción emocional } & 0.794 & 6.971 & 0.909 \\
\hline
\end{tabular}

* No estimado por haber sido utilizado para la identificación del modelo.

Fuente: Elaboración propia. 
En los apartados anteriores se hizo el análisis descriptivo de los tres aspectos evaluados, pero para realizar el análisis factorial confirmatorio solo se empleó el aspecto relativo a la satisfacción del visitante hacia los atractivos del destino turístico desde un punto de vista amplio. Esto se debió a dos razones: permite un análisis de los aspectos generales de su visita, y es la sección con pocos valores perdidos (en promedio $3.58 \%$ del total de encuestas) (cuadro 8).

Las cargas estandarizadas indican que la formación de variables latentes del factorial de primer orden están bien balanceadas (todas oscilan entre 1 y 0.4 ) y son significativas ( $\mathrm{p}<0.01$ ); no obstante, las de segundo orden tienen una carga factorial alta (0.841 y 0.794), aunque esta no es estadísticamente significativa ( $\mathrm{p}>0.01$ ), por lo que, desde la perspectiva de este trabajo, la muestra no permite inferir que exista una satisfacción bidimensional en los visitantes de cruceros encuestados.

El modelo planteado registra índices de bondad de ajuste satisfactorios (CFI $=0.975 ; \mathrm{TLI}=0.947 ; \mathrm{RMSEA}=0.142 ; \mathrm{SRMR}=0.025$ ), un índice comparativo de ajuste de Hu y Bentler (CFI) y un índice de Tucker-Lewis (TLI) mayores a 0.9, y el RMSEA y el SRMr menores a 0.05 son absolutamente deseables. Sin embargo, el SRMR cumple con el criterio pero el RMSEA no es el más deseable. En todo caso, la cantidad de la muestra analizada es amplia y los demás índices son aceptables. En la figura 1 se observan las cargas factoriales estandarizadas junto a su respectivo error estándar del modelo planteado.

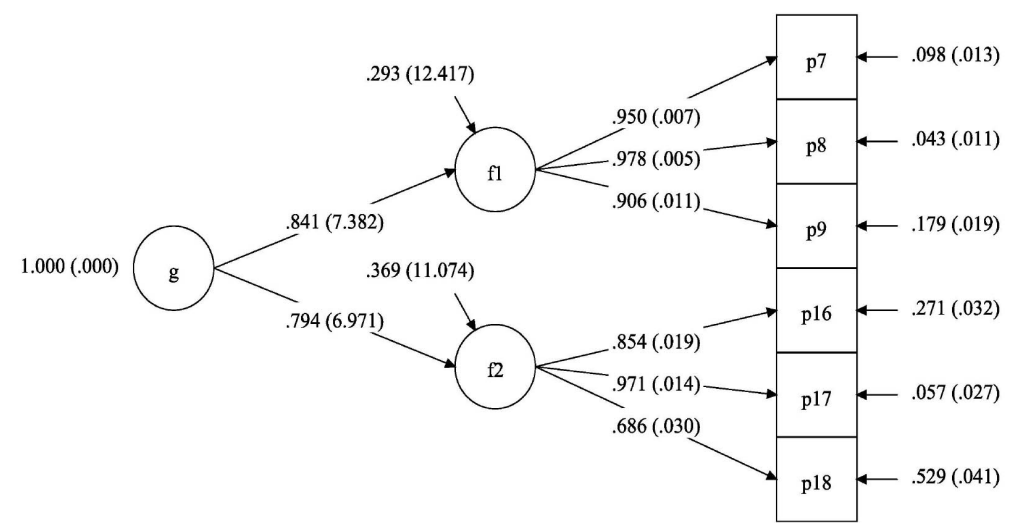

Fuente: Elaboración propia. 
Para calcular la media general o ponderada del grado de satisfacción emocional y evaluativa de los visitantes de cruceros se tomaron en consideración las cargas factoriales estandarizadas y los intercepts obtenidos de las corridas del MPlus, que corrigen las medias cuando existen datos faltantes, como en este caso. Los resultados se presentan en el cuadro 9.

Cuadro 9. Media ponderada de la satisfacción evaluativa y emocional

\begin{tabular}{lcclc} 
Satisfacción evaluativa & & & \\
Ítem & Intercepts & \multicolumn{1}{c}{$\lambda$ Std. } & Producto $\left({ }^{*} \lambda\right.$ Std. $)$ & $\begin{array}{c}\text { Media } \\
\text { ponderada }\end{array}$ \\
P7 & 7.096 & 0.950 & 6.7412 & \\
P8 & 6.848 & 0.978 & 6.697344 & 6.85 \\
P9 & 6.581 & 0.906 & 5.962386 & \\
& & $\sum 2.834$ & $\sum 19.40093$ & \\
Satisfacción emocional & & & Media \\
& & & & \\
& Intercepts & $\lambda$ Std. & Producto $\left({ }^{*} \lambda\right.$ Std.) & ponderada \\
P16 & 6.697 & 0.854 & 5.719238 & \\
P17 & 6.912 & 0.971 & 6.711552 & \\
P18 & 4.615 & 0.686 & 3.16589 & \\
& & $\sum 2.511$ & $\sum 15.59668$ &
\end{tabular}

Fuente: Elaboración propia.

En suma, se observa que, en una escala del uno al nueve, la media ponderada de la satisfacción del visitante de cruceros es 6.85 desde la perspectiva evaluativa y 6.21 de la emocional; esto revela que los visitantes de cruceros encuestados se encuentran suficientemente satisfechos con el destino en general. Es importante advertir que las cargas estandarizadas y las medias corregidas son más altas en la perspectiva de la satisfacción evaluativa que en la emocional. 


\section{Conclusiones}

Con fundamento en los resultados descriptivos de la escala evaluativa es posible establecer la falta de capacidad del visitante para evaluar los servicios de alimentación, debido a que no realizan este consumo durante su visita a la isla de Cozumel (20\% de los encuestados no contestaron ese apartado). Lo mismo se encontró en la medición de la escala emocional, donde de nueva cuenta se aprecia que $18.9 \%$ no emite opinión respecto de los servicios de alimentación. Estos datos, apoyados por las verbalizaciones de los encuestados, permiten sugerir que un porcentaje importante de los visitantes no utiliza servicios de alimentación durante su estadía en Cozumel.

En el caso de las aportaciones para decisiones gerenciales, se encontró que es posible evaluar la satisfacción de los visitantes a la isla de Cozumel desde alguna de estas perspectivas, y se sugiere realizarla desde la escala evaluativa, porque se vio que las cargas factoriales son más altas desde esta perspectiva, suponiendo que la satisfacción de estos visitantes hacia el destino pueda entenderse como un proceso evaluativo o cognitivo más que emocional, lo que sin duda contribuirá a la mejora continua de los diferentes establecimientos que ofrecen sus servicios a los visitantes.

Con esta aproximación a los análisis y con las características identificadas en cada escala se presenta la oportunidad de utilizarlas en investigaciones vinculadas con la satisfacción de visitantes que viajan hacia destinos de cruceros, en donde el periodo de visita es menor, por las peculiaridades del tipo de visitante que viaja en cruceros. Aunque la literatura sustenta la opción de concebir el análisis de la satisfacción como un constructo bidimensional (cognitivo-afectivo), en este trabajo no fue posible encontrar soporte empírico.

Adicionalmente es oportuno mencionar que este estudio tiene la limitante de la temporalidad de la aplicación del instrumento y la representatividad del muestreo, ya que fue aplicado en julio de 2013, que es cuando regularmente nuestro destino turístico registra las temperaturas más elevadas, acompañadas de periodos de mayor precipitación fluvial, lo cual puede considerarse un factor externo no controlado que puede afectar la satisfacción del visitante. En cuanto a la representatividad, tiene la limitante de haberse utilizado un muestreo no probabilístico, por lo que (si se busca obtener la perspectiva representada de los visitantes de cruceros en Cozumel) se sugiere que en futuras aplicaciones de este 
tipo de cuestionarios se realice esta aplicación de forma longitudinal y con un muestreo probabilístico del tipo por conglomerados o estratificado.

A pesar de las limitaciones, esta investigación es útil para tener una idea de la satisfacción emocional y evaluativa de los visitantes de cruceros en Cozumel, que, por lo demás, reportó resultados positivos y satisfactorios.

\section{Fuentes citadas}

Alegre, J. y J. Garau (2010). “Tourist Satisfaction and Dissatisfaction”. Annals of Tourism Research, 37 (1), 52-73.

Cestur (2012). Metodología del estudio de perfil y satisfacción del turista [en línea]. México: Centro de Estudios Superiores en Turismo-Secretaría de Turismo. Disponible en: http://ictur.sectur.gob.mx/pdf/estudioseinvestigacion/calidadycompetitividad/PST_formato2.pdf [28, junio, 2013].

Cronin, J., M. Brady y T. Hult (2000). “Assessing the Effects of Quality, Value, and Customer Satisfaction on Consumer Behavioral Intentions in Service Environments”. Journal of Retailing, 76 (2), 193-218.

Cronin, J. y S. Taylor (1992). "Measuring Service Quality: A Reexamination and Extension”. Journal of Marketing, 56 (3), 55-68.

Dirección General de Puertos-Secretaría de Comunicaciones y Transportes (2013). "Informe estadístico mensual. Movimiento de carga, buques y pasajeros. Enero-Diciembre, 2012-2013” [en línea]. México: Coordinación General de Puertos y Marina Mercante-Dirección General de PuertosSecretaría de Comunicaciones y Transportes. Disponible en: http:// www.sct.gob.mx/fileadmin/CGPMM/U_DGP/estadisticas/2013/ Mensuales/12_diciembre_2013.pdf [2014, 8 de marzo].

Evrard, Y. y P. Aurier (1994). "The Influence of Emotions on Satisfaction with Movie Consumption”. Journal of Satisfaction, Dissatisfaction and Complaining Behavior, 7, 119-125.

Femenía, O. (2011). La imagen de un destino turístico como herramienta del marketing [en línea]. Málaga: Universidad de Málaga. Disponible en: http://www.eumed.net/libros-gratis/2011c/986/destino \%20turistico \% 20como \% 20herramienta \% 20de\% 20marketing \% 20introduccion. htm [01, febrero, 2014]. 
Garbarino, E. y M. Johnson (1999). "The Different Roles of Satisfaction, Trust, and Commitment in Customer Relationships". Journal of Marketing, 63 (2), 70-87.

Hill, N., J. Brierley y R. MacDougall (2001). Cómo medir la satisfacción del cliente. México: Panorama Editorial.

Hoogland, J. y A. Boomsma (1998). "Robustness Studies in Covariance Structure Modeling: An Overview and a Meta-Analysis”. Sociological Methods and Research, 26 (3), 329-367.

Hoyle, R. (1995). Structural Equation Modeling: Concepts, Issues, and Applications. Thousand Oaks: Sage.

Izard, C. (1977). Human Emotions. Nueva York: Plenum Press.

Kozak, M. y M. Rimmington (2000). “Tourist Satisfaction with Mallorca, Spain, as an Off-Season Holiday Destination”. Journal of Travel Research, 38 (3), 260-269.

Latu, T. y A. Everett (2000). "Review of Satisfaction Research and Measurement Approaches” [en línea]. Wellington: Department of Conservation (Science and Research Internal Report, 183). Disponible en: http://www. doc.govt.nz/documents/science-and-technical/ir183.pdf

Muthén, L. y B. Muthén (2002). "How to Use a Monte Carlo Study to Decide on Sample Size and Determine Power”. Structural Equation Modeling, 9 (4), 599-620.

Nunnally, J. e I. Bernstein (1994). Psychometric Theory. 3a ed. Nueva York: McGraw Hill.

Oliver, R. (1997). Satisfaction: A Behavioral Perspective on the Consumer. Nueva York: McGraw-Hill.

Reynolds, P. y D. Braithwaite (2001). "Towards a Conceptual Framework for Wildlife Tourism”. Tourism Management, 22 (1), 31-42. Disponible en: http://www.sciencedirect.com/science/article/pii/S0261517700000182 Rodríguez, I. y H. San Martín (2008). “Tourist Satisfaction: A Cognitive-Affective Model”. Annals of Tourism Research, 35 (2), 551-573.

Sedetur (2013). "Indicadores Turísticos enero 2013” [en línea]. México: Secretaría de Turismo del Estado de Quintana Roo. Disponible en: http:// sedetur.qroo.gob.mx/estadisticas/indicadores/Indicadores \% 20Turisticos \%202013.pdf_[2014, 20 de mayo]. 
Swan, J. y F. Trawick (1993). “Consumer Satisfaction Research: 1983-1992 Accomplishments and Future Directions”. Journal of Consumer Satisfaction, Dissatisfaction and Complaining Behavior, 6, 28-33.

Westbrook, R. y R. Oliver (1991). “The Dimensionality of Consumption Emotion Patterns and Consumer Satisfaction”. Journal of Consumer Research, 18 (1), junio, 84-91.

Yu, L. y M. Goulden (2006). "A Comparative Analysis of International Tourist's Satisfaction in Mongolia”. Tourism Management, 27 (6), 1331-1342. 